\title{
A MULTIPLICATIVE INEQUALITY FOR CONCENTRATION FUNCTIONS OF $n$-FOLD CONVOLUTIONS
}

\author{
F. GötZe ${ }^{1}$ A. Yu. ZAitsev ${ }^{1,2}$ \\ University of Bielefeld ${ }^{1}$ \\ St. Petersburg Branch of Steklov Mathematical Institute ${ }^{2}$
}

December 1999

\begin{abstract}
We estimate the concentration functions of $n$-fold convolutions of one-dimensional probability measures. The main result is a supplement to the results of Götze and Zaitsev (1998). We show that the estimation of concentration functions at arguments of bounded size can be reduced to the estimation of these functions at arguments of size $O(\sqrt{n})$ which is easier.
\end{abstract}

\section{Introduction}

Let us first introduce some notation. Let $\mathfrak{F}$ denote the set of probability distributions defined on the Borel $\sigma$-field of subsets of the real line $\mathbf{R}, \mathcal{L}(\xi) \in \mathfrak{F}$ the distribution of a random variable $\xi$, and $\mathbf{I}\{A\}$ the indicator function of an event $A$. For $F \in \mathfrak{F}$ the concentration function is defined by $Q(F, b)=\sup _{x} F\{[x, x+b]\}, b \geqslant 0$. For $F, H \in \mathfrak{F}$ we denote the corresponding distribution functions by $F(x), H(x)$ and the characteristic functions by $\widehat{F}(t), \widehat{H}(t)$. Let $E_{a} \in \mathfrak{F}$ be the distribution concentrated at a point $a \in \mathbf{R}, E=E_{0}$. Products and powers of measures will be understood in the convolution sense: $F H=F * H, H^{m}=H^{m *}, H^{0}=E$. For $F=\mathcal{L}(\xi) \in \mathfrak{F}$ we shall use the notation $\bar{F}=\mathcal{L}(-\xi)$ and $\widetilde{F}=F \bar{F}$. The distribution $\widetilde{F}$ is called symmetrized. By $c(\cdot)$ we shall denote positive quantities depending on the arguments only. The symbol $c$ will be used for absolute positive constants.

1991 Mathematics Subject Classification. 60F05.

Key words and phrases. Concentration functions, sums of i.i.d. random variables, rates of decay.

${ }^{1}$ Research supported by the SFB 343 in Bielefeld.

${ }^{2}$ Research supported by Russian Foundation of Basic Research (RFBR) Grant 05-01-00911, by RFBR-DFG Grant 04-01-04000, and by the grant NSh 4222.2006.1

Typeset by $\mathcal{A} \mathcal{M S}-\mathrm{T}_{\mathrm{E}} \mathrm{X}$ 
Note that $c(\cdot), c$ can be different in different (or even in the same) formulas. The ends of proofs will be marked by $\square$.

We estimate the concentration functions of $n$-fold convolutions of one-dimensional probability measures. The concentration functions was introduced and preliminarily estimated by Lévy (1937), see as well Doeblin (1939). The bounds for these convolutions were obtained by Kolmogorov (1956, 1958), Rogozin (1961a,b), Le Cam (1965), Esseen (1966, 1968), Kesten (1969, 1972), Morozova (1977), Postnikova and Yudin (1978), Arak (1981), Miroshnikov and Rogozin (1980, 1982), Hall (1983), Griffin, Jain and Pruitt (1984), Arak and Zaitsev (1988), Nagaev and Khodzhabagyan (1996) and others.

The aim of this paper is to provide a supplement to a previous paper Götze and Zaitsev (1998) which is abbreviated in the following as GZ. We generalize Theorem 2 of that paper proving the following result.

Theorem 1. Assume that a distribution $F \in \mathfrak{F}$ is represented in the form

$$
F=(1-p) U+p V, \quad U, V \in \mathfrak{F}, \quad 0<p<1 .
$$

Let $X$ be a random variable with $\mathcal{L}(X)=U$. Suppose that

$$
0<\sigma^{2}=\mathbf{E} X^{2}<\infty, \quad \mathbf{E} X=0,
$$

and

$$
b \geqslant \frac{\varkappa_{n}}{\sigma^{2}}, \quad \text { where } \varkappa_{n}=\mathbf{E} X^{2} \min \{|X|, \sigma \sqrt{n}\} .
$$

Let $r, s$ be integers, $0 \leqslant r<s \leqslant n$. Then, for any distribution $H \in \mathfrak{F}$,

$$
\begin{aligned}
& Q\left(H F^{n}, b\right) \leqslant \mathbf{E} \frac{c b}{\sigma \sqrt{n-\mu}} Q\left(H V^{\mu}, \sigma \sqrt{n-\mu}\right) \mathbf{I}\{r \leqslant \mu<s\} \\
& \quad+\mathbf{E} Q\left(H U^{n-\mu} V^{\mu}, b\right) \mathbf{I}\{\mu \geqslant s\}+\min \left\{1, \frac{c b}{\sigma \sqrt{n-r}} Q(H, \sigma \sqrt{n})\right\} \mathbf{P}\{\mu<r\},
\end{aligned}
$$

where $\mu$ is a random variable having binomial distribution with parameters $n$ and $p$. Moreover,

$$
\begin{aligned}
Q\left(H F^{n}, b\right) \leqslant \frac{c b}{\sigma \sqrt{n(1-p)}} Q & \left.H V^{r}, \sigma \sqrt{n}\right) \\
& +\min \left\{1, \frac{c b}{\sigma \sqrt{n-r}} Q(H, \sigma \sqrt{n})\right\} \mathbf{P}\{\mu<r\} .
\end{aligned}
$$

Theorem 2 of GZ (1998) is a particular case of our Theorem 1 appearing when $H=E$. The moment restrictions in Theorem 1 are imposed on the distribution $U$ only. The distributions $H$ and $V$ are arbitrary and therefore the initial distribution $F$ may have arbitrarily bad moment properties.

Taking in (1.5) $r=0$, we obtain 
Corollary 1. Under the conditions of Theorem 1

$$
Q\left(H F^{n}, b\right) \leqslant \frac{c b}{\sigma \sqrt{n(1-p)}} Q(H, \sigma \sqrt{n}) .
$$

An important particular case of Theorem 1 and Corollary 1 appears when $n$ is changed by integer $n \alpha$ and $H=F^{n(1-\alpha)}$ where $0<\alpha<1$. Then the bound (1.6) can be rewritten in the form

$$
Q\left(F^{n}, b\right) \leqslant \frac{c b}{\sigma \sqrt{n \alpha(1-p)}} Q\left(F^{n(1-\alpha)}, \sigma \sqrt{n \alpha}\right) .
$$

Theorem 1 and Corollary 1 provide in a sense "multiplicative inequalities" for concentration functions of convolutions. The inequality (1.7) reduces the problem of estimation of $Q\left(F^{n}, b\right)$ to that of of $Q\left(F^{n(1-\alpha)}, \sigma \sqrt{n \alpha}\right)$. The trivial inequality

$$
Q\left(F^{n(1-\alpha)}, \sigma \sqrt{n \alpha}\right) \leqslant\left(1+\frac{\sigma \sqrt{n \alpha}}{b}\right) Q\left(F^{n(1-\alpha)}, b\right)
$$

(see (2.4)) together with (1.7) may return the problem to the initial stage (in particular, when $Q\left(F^{n}, b\right)$ decreases with some negative power of $\left.n\right)$. However, the inequality (1.8) may lead to a loss of precision. For instance, if $F$ is concentrated on a one-dimensional lattice with step size $2 \sigma \sqrt{n \alpha}$, we have

$$
Q\left(F^{n(1-\alpha)}, \sigma \sqrt{n \alpha}\right)=Q\left(F^{n(1-\alpha)}, b\right), \quad \text { for } \quad 0 \leqslant b \leqslant \sigma \sqrt{n \alpha} .
$$

It is clear that it is much easier to estimate the concentration function for the large value $\sigma \sqrt{n \alpha}$ of the argument than for some fixed $b$.

Applying Theorem 1 and Corollary 1, one can use known classical bounds for concentration functions. We mention in this connection the papers by Rogozin (1961a), Esseen (1968), Kesten (1969), Arak (1981), Miroshnikov and Rogozin (1980), Hall (1983), Griffin, Jain and Pruitt (1984). For example, the Esseen bound (1968), implies the following result.

Corollary 2. Let the conditions of Theorem 1 be satisfied. Then, for any $\delta>0$,

$$
Q\left(F^{n}, b\right) \leqslant \frac{c b(\delta+\sigma)}{\delta \sigma n \sqrt{(1-p) D(\widetilde{F}, \delta \sqrt{n})}} .
$$

where

$$
D(F, b)=\int_{-\infty}^{\infty} \min \left\{x^{2} b^{-2}, 1\right\} F\{d x\}, \quad F \in \mathfrak{F}, \quad b>0 .
$$

Esseen (1968) (see Petrov (1976), inequality (2.7) of Chapter III) proved that

$$
Q\left(F^{n}, b\right) \leqslant \frac{c}{\sqrt{n D(\widetilde{F}, b)}},
$$

Corollary 2 implies the following result. 
Corollary 3. Let $F \in \mathfrak{F}, b \geqslant 0, \delta>0$. Then

$$
Q\left(F^{n}, b\right) \leqslant \frac{c(F, b, \delta)}{n \sqrt{D(\widetilde{F}, \delta \sqrt{n})}} .
$$

For the proof it suffices to note that after a shift every non-degenerate distribution can be represented in the form (1.1) with $p=1 / 2$ and a non-degenerated $U$ having bounded support. This yields the result for $b \geqslant \varkappa / \sigma^{2}$, where $\varkappa=\mathbf{E}|X|^{3}$. For $b<\varkappa / \sigma^{2}$ we can apply the result for $b=\varkappa / \sigma^{2}$, using the monotonicity of concentration functions. Corollary 3 is Theorem 1 from the paper of GZ (1998) which is a sharpening of a result of Esseen (1968) who showed that $Q\left(F^{n}, b\right)=o\left(n^{-1 / 2}\right)$ as $n \rightarrow \infty$ iff the distribution $F$ has an infinite second moment (see as well Morozova (1977)). However the proof of Corollary 3 in the present paper is somewhat easier and we obtain here a more explicit form of $c(F, b, \delta)$. For the connection of Corollary 3 with previous results about concentration functions see GZ (1998).

Note that in the proof of Corollary 3 we use the inequality (1.11). Nevertheless, Corollary 3 can be considered as an improvement of the inequality (1.11). Indeed, the latter can be rewritten in the form

$$
Q\left(F^{n}, b\right) \leqslant c\left(n \int \min \left\{x^{2} b^{-2}, 1\right\} \widetilde{F}\{d x\}\right)^{-1 / 2}
$$

Comparing (1.12) with the inequality

$$
Q\left(F^{n}, b\right) \leqslant c(F, b)\left(n \int \min \left\{x^{2} b^{-2}, n\right\} \widetilde{F}\{d x\}\right)^{-1 / 2}
$$

which follows from Corollary 3 with $\delta=b$, we see that for any distributions with infinite variance the inequality (1.13) is sharper with respect to the order in $n$ than (1.12). Note however that it is impossible to change $c(F, b)$ in (1.13) by some absolute constant $c$. The corresponding example is given by the distribution $F=F_{n}=\frac{1}{2} E_{-n}+\frac{1}{2} E_{n}$. It is well known that $Q\left(F_{n}^{n}, 1\right)$ behaves as $O\left(n^{-1 / 2}\right)$ when $n \rightarrow \infty$. On the other hand,

$$
\left(n \int \min \left\{x^{2}, n\right\} \widetilde{F}_{n}\{d x\}\right)^{-1 / 2} \leqslant \frac{c}{n} .
$$

This implies that $c\left(F_{n}, 1\right) \geqslant c \sqrt{n}$ in (1.13).

Esseen (1968) proved that, for any $F \in \mathfrak{F}$ and $b>0$,

$$
Q(F, b) \leqslant c b \int_{|t| \leqslant b^{-1}}|\widehat{F}(t)| d t .
$$


Applying (1.14) to the distribution $F^{n}$, we obtain

$$
Q\left(F^{n}, b\right) \leqslant c b \int_{|t| \leqslant b^{-1}}|\widehat{F}(t)|^{n} d t .
$$

On the other hand, using (1.7) and then (1.14), we see that

$$
Q\left(F^{n}, b\right) \leqslant \frac{c b}{\sqrt{1-p}} \int_{|t| \sigma \sqrt{n \alpha} \leqslant 1}|\widehat{F}(t)|^{n(1-\alpha)} d t
$$

if $n \alpha$ is integer and $b \geqslant \mathbf{E}|X|^{3} / \sigma^{2}$. A comparison of (1.15) with (1.16) demonstrates an advantage of using the inequality (1.7).

Our proofs are based on non-uniform estimates in the Central Limit Theorem (CLT) and on elementary properties of concentration functions (see the proof of Lemma 1 in GZ (1998) and Zaitsev (1987, 1992)). In this respect our proofs differ from most of the previous papers, where Esseen's (1968) method of characteristic functions had been extensively used. One should note however that the CLT approach was applied in the seminal paper of Kolmogorov (1958). He used the uniform BerryEsseen bound in the CLT since non-uniform ones were not known at that time.

\section{Proofs}

Lemma 1 (GZ (1998, Lemma 3)). Let $\xi, \xi_{1}, \xi_{2}, \ldots, \xi_{n}$ be i.i.d. random variables, $\mathbf{E} \xi=0, W=\mathcal{L}\left(\xi_{1}+\cdots+\xi_{n}\right)$,

$$
B^{2}=n \mathbf{E} \xi^{2}>0, \quad \beta=n \mathbf{E} \xi^{2} \min \{|\xi|, B\}<\infty .
$$

and let $b \geqslant \frac{\beta}{B^{2}}$. Then, for any $G \in \mathfrak{F}$, we have

$$
Q(W G, b) \leqslant \frac{c b}{B} Q(G, B)
$$

Note that the inequality (2.2) is a particular case of the inequality (1.6) appearing when $p=0$.

We need the following well-known simple properties of concentration functions, which are valid for any $F, H \in \mathfrak{F}$ and $\gamma, \gamma_{1}, \gamma_{2}>0$ :

$$
\begin{gathered}
Q(F H, \gamma) \leqslant \min \{Q(F, \gamma), Q(H, \gamma)\} \\
Q\left(F, \gamma_{1}\right) \leqslant\left(1+\left\lceil\gamma_{1} / \gamma_{2}\right\rceil\right) Q\left(F, \gamma_{2}\right)
\end{gathered}
$$

where $\lceil\cdot\rceil$ is the integer part of a number (see, e.g., Hengartner and Theodorescu (1973)). 
Proof of Theorem 1. It is known that $H F^{n}$ can be written in the form

$$
H F^{n}=\mathcal{L}\left(S_{n}\right), \quad \text { where } \quad S_{n}=\zeta+\sum_{i=1}^{n}\left(1-\mu_{i}\right) \xi_{i}+\mu_{i} \eta_{i}
$$

and $\zeta, \xi_{i}, \eta_{i}, \mu_{i}$ are jointly independent random variables with

$$
\mathcal{L}(\zeta)=H, \quad \mathcal{L}\left(\xi_{i}\right)=U, \quad \mathcal{L}\left(\eta_{i}\right)=V, \quad \mathcal{L}\left(\mu_{i}\right)=(1-p) E+p E_{1} .
$$

Define $\mu=\sum_{i=1}^{n} \mu_{i}$. Obviously, $\mu$ has binomial distribution with parameters $n$ and $p$. Given a fixed value of $\mu$, the random variable $S_{n}$ has conditional distribution $H U^{n-\mu} V^{\mu}$. Hence, for any $x \in \mathbf{R}$ we have

$$
\begin{aligned}
\mathbf{P}\left\{S_{n} \in[x, x+b]\right\} & =\mathbf{E} \mathbf{I}\left\{S_{n} \in[x, x+b]\right\} \\
& =\mathbf{E} \mathbf{E}\left\{\mathbf{I}\left\{S_{n} \in[x, x+b]\right\} \mid \mu\right\} \\
& \leqslant \mathbf{E} Q\left(H U^{n-\mu} V^{\mu}, b\right) .
\end{aligned}
$$

Therefore,

$$
Q\left(H F^{n}, b\right) \leqslant \mathbf{E} Q\left(H U^{n-\mu} V^{\mu}, b\right) .
$$

In view of $\varkappa_{n-\mu} \leqslant \varkappa_{n}$ and applying inequality (2.2) of Lemma 1 with $W=U^{n-\mu}$, $G=H V^{\mu}, B=\sigma \sqrt{n-\mu}$, we see that

$$
\begin{aligned}
& \mathbf{E} Q\left(H U^{n-\mu} V^{\mu}, b\right) \leqslant \mathbf{E} \frac{c b}{\sigma \sqrt{n-\mu}} Q\left(H V^{\mu}, \sigma \sqrt{n-\mu}\right) \mathbf{I}\{r \leqslant \mu<s\} \\
& +\mathbf{E} Q\left(H U^{n-\mu} V^{\mu}, b\right) \mathbf{I}\{\mu \geqslant s\}+\mathbf{E} Q\left(H U^{n-\mu} V^{\mu}, b\right) \mathbf{I}\{\mu<r\}
\end{aligned}
$$

and

$$
\mathbf{E} Q\left(H U^{n-\mu} V^{\mu}, b\right) \mathbf{I}\{\mu<r\} \leqslant \min \left\{1, \frac{c b}{\sigma \sqrt{n-r}} Q(H, \sigma \sqrt{n})\right\} \mathbf{P}\{\mu<r\} .
$$

The inequality (1.4) now follows from (2.5)-(2.7).

Using the relations $\mathbf{P}\{\mu=n\}=p^{n}$, (1.4) with $s=n$ and (2.3), we obtain

$$
\begin{aligned}
Q\left(H F^{n}, b\right) \leqslant & \frac{c b}{\sigma} Q\left(H V^{r}, \sigma \sqrt{n}\right) \mathbf{E} \frac{1}{\sqrt{n-\mu}} \mathbf{I}\{r \leqslant \mu<n\} \\
& +p^{n} Q\left(H V^{n}, b\right)+\min \left\{1, \frac{c b}{\sigma \sqrt{n-r}} Q(H, \sigma \sqrt{n})\right\} \mathbf{P}\{\mu<r\} .
\end{aligned}
$$

Applying the Hölder inequality, we derive

$$
\begin{aligned}
\mathbf{E} \frac{1}{\sqrt{n-\mu}} \mathbf{I}\{r \leqslant \mu<n\} & \leqslant c \mathbf{E} \frac{1}{\sqrt{n-\mu+1}} \\
& \leqslant c\left(\mathbf{E} \frac{1}{n-\mu+1}\right)^{1 / 2} \leqslant \frac{c}{\sqrt{n(1-p)}} .
\end{aligned}
$$


Using (1.2) and (1.3), it is easy to see that

$$
\begin{aligned}
\varkappa_{n} & \geqslant \varkappa_{1}=\mathbf{E}|X|^{3} \mathbf{I}\{|X| \leqslant \sigma\}+\sigma \mathbf{E}|X|^{2} \mathbf{I}\{|X|>\sigma\} \\
& \geqslant\left(\mathbf{E}|X|^{2} \mathbf{I}\{|X| \leqslant \sigma\}\right)^{3 / 2}+\left(\mathbf{E}|X|^{2} \mathbf{I}\{|X|>\sigma\}\right)^{3 / 2} \geqslant c \sigma^{3} .
\end{aligned}
$$

The relations (1.3) and (2.10) together imply

$$
b \geqslant \varkappa_{n} \sigma^{-2} \geqslant c \sigma
$$

According to (2.3), (2.4) and (2.11), we have

$$
\begin{aligned}
Q\left(H V^{n}, b\right) \leqslant Q\left(H V^{r}, b\right) & \leqslant\left(\frac{b}{\sigma \sqrt{n}}+1\right) Q\left(H V^{r}, \sigma \sqrt{n}\right) \\
& \leqslant \frac{c b}{\sigma} Q\left(H V^{r}, \sigma \sqrt{n}\right)
\end{aligned}
$$

Moreover,

$$
p^{n}=(1-(1-p))^{n} \leqslant e^{-n(1-p)} \leqslant \frac{c}{\sqrt{n(1-p)}} .
$$

The inequality (1.5) can be easily derived from (2.8), (2.9), (2.12) and (2.13).

Proof of Corollary 2. In view of (2.3), (2.11), we may assume without loss of generality that $n$ is even and $n \geqslant 2$. Using (1.7) with $\alpha=1 / 2,(1.11),(2.3)$ and (2.4), we obtain

$$
\begin{aligned}
Q\left(F^{n}, b\right) & \leqslant \frac{c b}{\sigma \sqrt{n(1-p)}} Q\left(F^{n / 2}, \sigma \sqrt{n}\right) \\
& \leqslant \frac{c b}{\sigma \sqrt{n(1-p)}}\left(1+\sigma \delta^{-1}\right) Q\left(F^{n / 2}, \delta \sqrt{n}\right) \\
& \leqslant \frac{c b}{\sigma \sqrt{n(1-p)}} \frac{1+\sigma \delta^{-1}}{\sqrt{n D(\widetilde{F}, \delta \sqrt{n})}} \\
& =\frac{c b(\delta+\sigma)}{\delta \sigma n \sqrt{(1-p) D(\widetilde{F}, \delta \sqrt{n})}} \cdot
\end{aligned}
$$

\section{REFERENCES}

Arak, T. V., On the convergence rate in Kolmogorov's uniform limit theorem. I, Theory Probab. Appl. 26 (1981), no. 2, 219-239.

Arak, T. V., and Zaitsev, A. Yu., Uniform limit theorems for sums of independent random variables, Proc. Steklov Inst. Math. 174 (1988).

Doeblin, W., Sur les sommes d'un grand nombre des variables aléatoire independantes, Bull Sci. Math. 63 (1939), 23-32; 35-64. 
Esseen, C.-G., On the Kolmogorov-Rogozin inequality for the concentration function, Z. Wahrscheinlichkeitstheor. verw. Geb. 5 (1966), 210-216.

Esseen, C.-G., On the concentration function of a sum of independent random variables, Z. Wahrscheinlichkeitstheor. verw. Geb. 9 (1968), no. 4, 290-308.

Götze, F., and Zaitsev, A. Yu., Estimates for the rapid decay of concentration functions of $n$-fold convolutions, J. Theoret. Probab. 11 (1998), no. 3, 715-731.

Griffin, P. S., Jain, N. C., and Pruitt, W. E., Approximate local limit theorems outside domains of attraction, Ann. Probab. 12 (1984), no. 1, 45-63.

Hall, P., Order of magnitude of the concentration function, Proc. Amer. Math. Soc. 89 (1983), no. $1,141-144$.

Hengartner, W., and Theodorescu, R., Concentration functions, Academic Press, New York, 1973.

Kesten, H., A sharper form of the Doeblin-Lévy-Kolmogorov-Rogozin inequality for concentration functions, Math. Scand. 25 (1969), 133-144.

Kesten, H., Sums of independent random variables-without moment conditions, Ann. Math. Statist. 24 (1972), 701-732.

Kolmogorov, A. N., Two uniform limit theorems for sums of independent random variables, Theory Probab. Appl. 1 (1956), no. 4, 384-394.

Kolmogorov, A., Sur les propriétés des fonctions de concentrations de M. P. Lévy, Ann. Inst. H. Poincaré 16 (1958), 27-34.

Le Cam, L., On the distribution of sums of independent random variables, In: Bernoulli, Bayes, Laplace (anniversary volume), Springer, Berlin, 1965, pp. 179-202.

Lévy, P., Théory de l'addition des variables aléatoires, Gauthier-Villar, Paris, 1937.

Miroshnikov, A. L., and Rogozin, B. A., Inequalities for concentration functions, Theor. Probab. Appl. 25 (1980), no. 1, 176-180.

Miroshnikov, A. L., and Rogozin, B. A., Remarks on an inequality for the concentration function of sums of independent variables, Theor. Probab. Appl. 27 (1982), no. 4, 848-850.

Morozova, L. N., Some bounds for the concentration functions of a sum of independent and identically distributed random variables, In: Limit Theorems for Random Processes (1977), Fan, Tashkent, 85-91. (Russian)

Nagaev, S. V., and Khodzhabagyan, S. S., On an estimate for the concentration function of sums of independent random variables, Theor. Probab. Appl. 41 (1996), no. 3, 560-569.

Petrov, V. V., Limit theorems for sums of independent random variables, Springer, Berlin, 1976.

Rogozin, B. A., An estimate for concentration functions, Theory Probab. Appl. 6 (1961a), no. 1, 94-97.

Rogozin, B. A., On the increase of dispersion of sums of independent random variables, Theory Probab. Appl. 6 (1961b), no. 1, 97-99.

Postnikova, L. P., Yudin, A. A., A sharper form of the inequality for the concentration function, Theory Probab. Appl. 23 (1978), no. 2, 359-362.

Zaitsev, A. Yu., On the uniform approximation of distributions of sums of independent random variables, Theory Probab. Appl. 32 (1987), no. 1, 40-47.

Zaitsev, A. Yu., Approximation of convolutions of probability distributions by infinitely divisible laws under weakened moment restrictions, Zapiski Nauchnykh Seminarov LOMI 194 (1992), 79-90 (Russian); English transl. in J. Math. Sci. 75 (1995), no. 5, 1992-1930. 
Friedrich Götze

FAKUltät FÜR MAThematiK

Universität Bielefeld

Postfach 100131

33501 BielefEld 1

Germany

E-mail address: goetze@mathematik.uni-bielefeld.de

Andrei Yu. Zaitsev

St. Petersburg Branch of Steklov Mathematical Institute

FONTANKA 27

St. Petersburg 191011

Russia

E-mail address: zaitsev@pdmi.ras.ru 